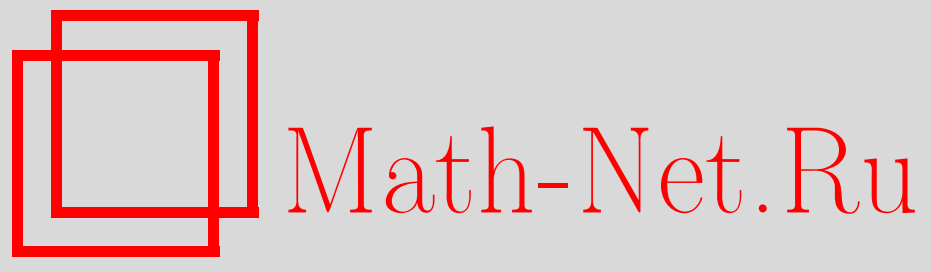

Д. Руджери, Квантовые каналы с памятью, распределенной по закону Гаусса, ТМФ, 2007, том 152, номер 2, 390-404

DOI: https://doi.org/10.4213/tmf6095

Использование Общероссийского математического портала Math-Net.Ru подразумевает, что вы прочитали и согласны с пользовательским соглашением http://www . mathnet.ru/rus/agreement

Параметры загрузки:

IP : 54.197 .130 .99

26 апреля 2023 г., 16:36:24

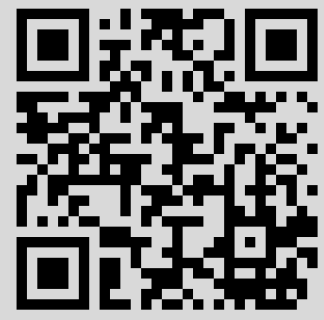




\section{КВАНТОВЫЕ КАНАЛЫ С ПАМЯТЬЮ, РАСПРЕДЕЛЕННОЙ ПО ЗАКОНУ ГАУССА}

Исследуется процесс передачи информации в рамках двух различных моделей гауссово распределенной памяти в канале передачи: в канале с аддитивной гауссовой памятью и в канале с бозонной памятью с потерями. Показано, что зацепление начальных данных позволяет улучшить скорость передачи информации в указанных каналах.

Ключевые слова: квантовая передача информации, зацепление, неклассические состояния.

\section{1. ВВЕДЕНИЕ}

Передача информации требует кодирования информации в виде физической системы. Тем самым законы физики налагают ограничения на передачу и обработку информации. Известно, что любая обработка квантовой информации может быть представлена с помощью квантового канала $T$. До настоящего времени квантовая передача информации по каналам с шумами в основном рассматривалась для каналов без памяти [1], которые характеризуются тем, что шум влияет независимо на каждый символ, передающийся по каналу. Однако во многих реальных приложениях предположение об отсутствии шума в канале не выполняется и необходимо принимать в расчет эффекты памяти. Вследствие этого в последнее время наблюдается повышенный интерес к квантовым каналам с эффектами памяти, которые были названы каналами с памятью [2]-[4]. Каналы с памятью характеризуются наличием коррелированного источника шума, в присутствии которого каждое использование канала затронуто предыдущим использованием. Моделируя реальный шум с помощью простых моделей, можно достичь максимальной скорости передачи информации [5].

Исследования таких систем с использованием дискретных алфавитов (кубитов) были проведены в работе [3], где было отмечено, что зацепленные коды могут быть

${ }^{*}$ Dipartimento di Fisica, Università del Salento, I-73100 Lecce, Italy. E-mail: Giovanna.Ruggeri@le.infn.it 
полезны для достижения оптимальной работы канала. Затем некоторые из результатов этой работы были обобщены на случай непрерывных переменных в работах [6]-[8], а систематический анализ этой задачи был предложен в работе [9].

В данной работе исследуется процесс передачи информации с помощью каналов с непрерывной памятью [10], [11], таких как бозонные каналы и в более общем случае гауссовы каналы, моделированные с помощью моды бозонного поля, квадратуры фазового пространства которого применяются для кодирования и декодирования непрерывной переменной. Типичными примерами являются свободное пространство или передача сигнала по оптическому волокну, когда для передачи информации используются фотоны. Более того, эффекты памяти моделируются путем рассмотрения корреляций между действиями окружения при последовательных использованиях канала [6].

В разделе 2 исследуется модель для квантового гауссового канала, для которой Холево и др. [10], [11] явно вычислили классическую передающую способность. Используя этот результат в качестве исходного, мы исследуем поведение качества передачи информации в двух различных схемах гауссовых каналов [7], [8]. В разделе 3 рассматривается аддитивный гауссов канал [7], в котором входные данные дополнены тепловым шумом, что обобщает модель, представленную в [12]. Затем в разделе 4 исследуется бозонный канал с потерями, который состоит из набора бозонных мод, в которых энергия теряется по пути от передатчика к приемнику [13]. В нашем случае потери моделируются с помощью разделителя пучков [8]. Численные расчеты в этих моделях показывают, что с помощью зацепления удается улучшить качество передачи информации. Наконец, в разделе 5 содержатся заключительные замечания.

\section{2. КЛАССИЧЕСКАЯ ПЕРЕДАТОЧНАЯ СПОСОБНОСТЬ КВАНТОВЫХ ГАУССОВЫХ КАНАЛОВ}

Рассмотрим квантовую систему, заданную операторами уничтожения $\hat{a}_{1}, \ldots, \hat{a}_{n}$, удовлетворяющими каноническим коммутационным соотношениям:

$$
\left[\hat{a}_{j}, \hat{a}_{k}^{\dagger}\right]=\delta_{j k}, \quad\left[\hat{a}_{j}, \hat{a}_{k}\right]=0
$$

или, что эквивалентно, соответствующими операторами координаты и импульса $\hat{x}_{1}, \ldots, \hat{x}_{n}$ и $\hat{p}_{1}, \ldots, \hat{p}_{n}$. Введем при этом вещественнозначные $2 n$-компонентные вектор-столбцы

$$
\mathbf{r}=\left[\hat{x}_{1}, \ldots, \hat{x}_{n}, \hat{p}_{1}, \ldots, \hat{p}_{n}\right]^{\mathrm{T}}, \quad \mathbf{z}=\left[y_{1}, \ldots, y_{n}, q_{1}, \ldots, q_{n}\right]^{\mathrm{T}}
$$

и унитарные операторы

$$
\widehat{V}(\mathbf{z})=e^{i \mathbf{r}^{\mathrm{T}} \mathbf{z}}
$$

удовлетворяющие коммутационным соотношениям Сигала-Вейля

$$
\widehat{V}(\mathbf{z}) \widehat{V}\left(\mathbf{z}^{\prime}\right)=e^{i \mathcal{J}\left(\mathbf{z}, \mathbf{z}^{\prime}\right) / 2} \widehat{V}\left(\mathbf{z}+\mathbf{z}^{\prime}\right),
$$


с канонической симплектической формой

$$
\mathcal{J}\left(\mathbf{z}, \mathbf{z}^{\prime}\right)=\sum_{j=1}^{n}\left(y_{j}^{\prime} q_{j}-y_{j} q_{j}^{\prime}\right)=\mathbf{z}^{\prime \mathrm{T}} \mathcal{J} \mathbf{z},
$$

где $\mathcal{J}$ - кососимметричная $(2 n \times 2 n)$-матрица

$$
\mathcal{J}=\left(\begin{array}{cc}
0 & \mathcal{I} \\
-\mathcal{I} & 0
\end{array}\right)
$$

a $\mathcal{I}$ - единичная матрица размера $n \times n$.

Оператор плотности $\hat{\rho}$ называется гауссовым, если его квантовая характеристическая функция $\phi(\mathbf{z})=\operatorname{Tr}(\hat{\rho} \widehat{V}(\mathbf{z}))$ имеет вид

$$
\phi(\mathbf{z})=\exp \left(i \mathbf{m}^{\mathrm{T}} \mathbf{z}-\frac{1}{2} \mathbf{z}^{\mathrm{T}} \mathcal{V} \mathbf{z}\right),
$$

где $\mathbf{m}-2 n$-компонентный вектор-столбец, а $\mathcal{V}$ - вещественнозначная симметричная матрица размера $2 n \times 2 n$. Можно показать, что

$$
\mathbf{m}=\operatorname{Tr}(\hat{\rho} \mathbf{r}), \quad \mathcal{V}=\operatorname{Tr}\left(\mathbf{r} \hat{\rho} \mathbf{r}^{\mathrm{T}}\right)-\frac{i}{2} \mathcal{J}
$$

т.е. $\mathbf{m}$ - усредненный вектор, а $\mathcal{V}$ - корреляционная матрица, которая должна удовлетворять обобщенному соотношению неопределенностей Робертсона:

$$
\mathcal{V}-\frac{i}{2} \mathcal{J} \geqslant 0
$$

Ранее было доказано [14], что для произвольной вещественнозначной симметричной матрицы $\mathcal{V}$ существует линейное преобразование, с помощью которого можно получить разложение по ортогональным модам оператора плотности:

$$
\hat{\rho}=\bigotimes_{j=1}^{n} \hat{\rho}^{(j)} .
$$

Таким образом, вследствие такого разложения можно выписать энтропию фон Неймана гауссова состояния в терминах энтропии фон Неймана для каждой отдельной моды:

$$
S(\hat{\rho})=\sum_{j=1}^{n} S\left(\hat{\rho}^{(j)}\right) .
$$

Более того, проводя сравнение между отдельной модой гауссова состояния и калибровочно-инвариантным состоянием (см. [10], [11]), получим окончательное решение для энтропии фон Неймана:

$$
S(\hat{\rho})=\sum_{j=1}^{n} g\left(\left|\lambda_{j}\right|-\frac{1}{2}\right),
$$


в котором функция $g(x)$ представляет собой энтропию теплового состояния (со средним числом фотонов $x)$, задаваемую формулой

$$
g(x)= \begin{cases}(x+1) \ln (x+1)-x \ln x, & x>0 \\ 0, & x=0\end{cases}
$$

a $\pm \lambda_{j}$ суть симплектические собственные значения матрицы $\mathcal{V}$, которые являются решениями уравнения

$$
\operatorname{det}[\mathcal{V}-i \lambda \mathcal{J}]=0
$$

Рассмотрим процесс классического использования бозонного канала, описываемый полностью положительным и сохраняющим след отображением $T: \rho \mapsto T[\rho]$. Тогда квантовым состояниям отвечают значения случайной классической величины. В общем случае можно считать, что точки фазового пространства отображаются в квантовые состояния, а потому каждой моде ставятся в соответствие две вещественные случайные величины (или одна комплексная). При этом входное состояние $\rho_{\boldsymbol{\mu}}$ над $n$ модами (использованиями) нумеруется посредством индекса $\boldsymbol{\mu} \in \mathbb{C}^{n}$.

Теорема кодирования для квантовых каналов утверждает, что классическая передающая способность бозонного канала $T$ задается формулой

$$
C(T)=\lim _{n \rightarrow \infty} \frac{1}{n} \max \left[S\left(\int P\left(d^{2 n} \boldsymbol{\mu}\right) T\left[\rho_{\boldsymbol{\mu}}\right]\right)-\int P\left(d^{2 n} \boldsymbol{\mu}\right) S\left(T\left[\rho_{\boldsymbol{\mu}}\right]\right)\right],
$$

где $P\left(d^{2 n} \boldsymbol{\mu}\right)$ - вероятностная мера. В выражении (15) максимум берется по всем вероятностным мерам $\left\{P\left(d^{2 n} \boldsymbol{\mu}\right)\right\}$ и наборам операторов плотности $\left\{\rho_{\boldsymbol{\mu}}\right\}$, удовлетворяющим ограничениям по энергии:

$$
\frac{1}{n} \int P\left(d^{2 n} \boldsymbol{\mu}\right) \operatorname{Tr}\left(\rho_{\boldsymbol{\mu}} \sum_{j=1}^{n} a_{j}^{\dagger} a_{j}\right) \leqslant \bar{n}
$$

где $\bar{n}$ - максимальное среднее число фотонов на моду на входе канала.

\section{3. АДДИТИВНЫЙ ГАУССОВ КАНАЛ}

Рассмотрим обобщение модели, предложенной в [12], которое можно использовать при произвольном числе использований канала. Эффект памяти, как и в работе [7], моделируется с помощью предположения, что шум, влияющий на последующие использования канала, удовлетворяет распределению Гаусса с корреляцией за счет смежных двухмодовых взаимодействий.

Рассмотрим теперь отдельный бозонный канал $T$, действие которого имеет вид

$$
\hat{\rho}_{\boldsymbol{\mu}}^{\text {out }} \equiv T\left[\hat{\rho}_{\boldsymbol{\mu}}^{\text {in }}\right]=\int d^{2 n} \boldsymbol{\nu} Q(\boldsymbol{\nu}) \widehat{D}(\boldsymbol{\nu}) \hat{\rho}_{\boldsymbol{\mu}}^{\text {in }} \widehat{D}^{\dagger}(\boldsymbol{\nu}),
$$

где через $\widehat{D}(\boldsymbol{\nu}) \equiv \widehat{D}_{a_{1}}\left(\nu_{1}\right) \ldots \widehat{D}_{a_{n}}\left(\nu_{n}\right), \widehat{D}_{a_{j}}\left(\nu_{j}\right)=\exp \left(\nu_{j} \hat{a}_{j}^{\dagger}-\nu_{j}^{*} \hat{a}_{j}\right)$, обозначается оператор перемещения, действующий на $j$-ю моду, и $d^{2 n} \boldsymbol{\nu} \equiv d^{2} \nu_{1} d^{2} \nu_{2} \ldots d^{2} \nu_{n}, \nu_{j} \in \mathbb{C}$. 
Этот канал известен как аддитивный гауссов канал, если ядро преобразования представляет собой гауссову экспоненту, т.е.

$$
Q(\boldsymbol{\nu})=\prod_{j=1}^{n} \frac{1}{\pi N} e^{-\left|\nu_{j}\right|^{2} / N} .
$$

В таком случае канал случайным образом перемещает каждое входное когерентное состояние в соответствии с распределением Гаусса, что приводит к тепловому состоянию $\left(N\right.$ - дисперсия добавленного шума на квадратурных компонентах $\hat{x}_{j}$ и $\hat{p}_{j}$, или, что эквивалентно, число тепловых фотонов, добавляемых каналом к каждой отдельной моде).

Поскольку для гауссовых каналов предполагается, что гауссовы входные данные позволяют достичь наилучшей передачи информации [10], [11], будем кодировать $\boldsymbol{\mu}$ с помощью когерентных входных состояний

$$
\rho_{\boldsymbol{\mu}}^{\text {in }}=D(\boldsymbol{\mu})|\mathbf{0}\rangle\langle\mathbf{0}| D^{\dagger}(\boldsymbol{\mu}),
$$

где $|\mathbf{0}\rangle \equiv|0\rangle|0\rangle \ldots|0\rangle$ - вакуумные состояния $n$ указанных мод. Более того, предположим, что состояние (19) выпадает с вероятностью

$$
P(\boldsymbol{\mu})=\prod_{j=1}^{n} \frac{1}{\pi \bar{n}} e^{-\left|\mu_{j}\right|^{2} / \bar{n}},
$$

тогда усредненное выходное состояние имеет вид

$$
\bar{\rho}^{\text {out }}=\int d^{2 n} \boldsymbol{\mu} P(\boldsymbol{\mu}) \hat{\rho}_{\boldsymbol{\mu}}^{\text {out }} .
$$

Гауссово отображение, задаваемое аддитивным гауссовым каналом (17), в случае гауссова входного сигнала полностью задается своими ковариационными матрицами, т.е.

$$
\mathcal{V}^{\text {in }} \mapsto \mathcal{V}^{\text {in }}+\operatorname{diag}(N, N, \ldots, N),
$$

где $\mathcal{V}^{\text {in }}$ - ковариационная матрица на входе канала. Для состояний (19) она имеет вид

$$
\mathcal{V}^{\text {in }}=\frac{1}{2} \operatorname{diag}(1,1, \ldots, 1) .
$$

Следует также рассмотреть возможность зацепленных входных состояний. Это можно сделать с помощью преобразования $\hat{\rho}_{\boldsymbol{\mu}}^{\text {in }} \rightarrow \Sigma(\mathcal{R}) \hat{\rho}_{\boldsymbol{\mu}}^{\text {in }} \Sigma^{\dagger}(\mathcal{R})$, которое использует оператор многомодового сжатия [15]

$$
\Sigma(\mathcal{R})=\exp \left[\frac{1}{2} \sum_{\substack{j, k=1 \\ j \neq k}}^{n}\left(\mathcal{R}_{j k} \hat{a}_{j}^{\dagger} \hat{a}_{k}^{\dagger}-\mathcal{R}_{j k}^{*} \hat{a}_{j} \hat{a}_{k}\right)\right]
$$

где $\mathcal{R}$ - симметричная матрица размера $n \times n$. Таким образом, ковариационная матрица на входе имеет вид

$$
\mathcal{V}^{\text {in }}=\frac{1}{2}\left(\begin{array}{cc}
e^{\mathcal{R}} & 0 \\
0 & e^{-\mathcal{R}}
\end{array}\right)
$$


Пусть $\bar{n}_{r}-$ среднее число сжатых фотонов на моду, добавленных на входе, т.е.

$$
\bar{n}_{r}=\frac{1}{n} \sum_{j=1}^{n}\left\langle\mathbf{0}\left|\Sigma(\mathcal{R}) \hat{a}_{j}^{\dagger} \hat{a}_{j} \Sigma^{\dagger}(\mathcal{R})\right| \mathbf{0}\right\rangle .
$$

Тогда в силу ограничения (16) эффективное число фотонов, добавляемых на входе к отдельной моде, имеет вид $\bar{n}-\bar{n}_{r}$.

Опишем теперь эффекты памяти в аддитивном гауссовом канале. Выходные состояния (17) можно рассматривать как свертку входного гауссова состояния (19) с гауссовым тепловым состоянием, содержащим в среднем $N$ тепловых фотонов на моду. Чтобы ввести корреляции для этих состояний, можно использовать аргументацию, использованную в случае многомодового сжатия. Физически оправданно, что лишь последовательные использования канала могут быть сильно коррелированны. Введем матрицу $\mathcal{M}$ взаимодействия памяти, все элементы которой равны нулю, кроме элементов на двух побочных диагоналях, которые равны единице, т.е. $\mathcal{M}_{j-1, j}=M_{j, j-1}=1, j=2,3, \ldots, 2 n$. Положим теперь

$$
\mathcal{S}=-s \mathcal{M}
$$

где $s$ - параметр, измеряющий степень памяти. Выберем при этом добавленный шум, задаваемый ковариационной матрицей, в виде

$$
\mathcal{V}^{N}=\mathcal{V}_{1}^{N}+\epsilon \mathcal{V}_{2}^{N}
$$

где $\mathcal{V}_{1}^{N}$ - диагональная матрица

$$
\left[\mathcal{V}_{1}^{N}\right]_{j j}=N-\epsilon\left[\mathcal{V}_{2}^{N}\right]_{j j}
$$

a

$$
\mathcal{V}_{2}^{N}=\frac{1}{2}\left(\begin{array}{cc}
e^{\mathcal{S}} & 0 \\
0 & e^{-\mathcal{S}}
\end{array}\right) .
$$

Для построения физического преобразования мы должны потребовать, чтобы в канале выполнялось условие $\mathcal{V}^{N} \geqslant 0$. Очевидно, оно выполнено, если $\mathcal{V}_{1}^{N} \geqslant 0$ и $\mathcal{V}_{2}^{N} \geqslant 0$. Первое условие задает область разрешенных значений параметра памяти $s$, а второе условие выполнено всегда. Присутствие параметра $\epsilon$ в формуле (28) представляет собой всего лишь математический прием, обеспечивающий положительность $\mathcal{V}^{N}$ даже в случае, когда $N<1 / 2$. Он определяется следующим образом:

$$
\begin{gathered}
\epsilon=1, \quad N \geqslant \frac{1}{2}, \\
0 \leqslant \epsilon \leqslant 2 N, \quad N<\frac{1}{2} .
\end{gathered}
$$

Заметим, что введенные таким образом шумовые корреляции оказываются классическими, и в пределе $s \rightarrow 0$ в (28) восстанавливается случай с отсутствием памяти. 
Для зацепленных входных данных в выражении (24) в качестве симметричной матрицы $\mathcal{R}$ используем заданную в (27) матрицу $\mathcal{S}$ с заменой $s \rightarrow r$, где $r$ - параметр зацепления состояний на входе. На выходе канала мы при этом получим ковариационную матрицу

$$
\mathcal{V}^{\text {out }}=\mathcal{V}^{\text {in }}+\mathcal{V}^{N}
$$

В свою очередь, ковариационная матрица, связанная со смесью состояний на выходе (21), имеет вид

$$
\overline{\mathcal{V}}^{\text {out }}=\mathcal{V}^{\text {out }}+\mathcal{K}
$$

где

$$
\mathcal{K}=\mathcal{K}_{1}+\theta \mathcal{K}_{2}
$$

$\theta$ - параметр, $\mathcal{K}_{1}$ - диагональная матрица,

$$
\left[\mathcal{K}_{1}\right]_{j j}=\left(\bar{n}-\bar{n}_{r}\right)-\theta\left[\mathcal{K}_{2}\right]_{j j}
$$

и $\mathcal{K}_{2}$ снова имеет вид $\mathcal{V}_{2}^{N}$ при замене $s \rightarrow-y$. Коэффициент $y$ ответствен за возможные корреляции между классическими входными состояниями.

Также в указанном случае должно выполняться условие $\mathcal{K} \geqslant 0$. Очевидно, оно выполнено, если $\mathcal{K}_{1} \geqslant 0$ и $\mathcal{K}_{2} \geqslant 0$. Первое из неравенств задает область разрешенных значений параметра $y$, а второе выполнено всегда. Присутствие параметра $\theta$ в (34) оправданно лишь как математический прием, позволяющий обеспечить положительность $\mathcal{K}$ даже в случае, когда $\left(\bar{n}-\bar{n}_{r}\right)<1 / 2$. Он определяется следующим образом:

$$
\begin{gathered}
\theta=1, \quad \bar{n}-\bar{n}_{r} \geqslant \frac{1}{2}, \\
0 \leqslant \theta \leqslant 2\left(\bar{n}-\bar{n}_{r}\right), \quad \bar{n}-\bar{n}_{r}<\frac{1}{2} .
\end{gathered}
$$

В соответствии с выражением (15) передающая способность представляет собой максимум по коэффициенту передачи информации

$$
R=\frac{1}{n}\left[S\left(\bar{\rho}^{\text {out }}\right)-S\left(\hat{\rho}^{\text {out }}\right)\right] .
$$

Так как $\bar{\rho}^{\text {out }}$ и $\hat{\rho}^{\text {out }}$ суть гауссовы состояния, то с учетом (12) получим

$$
R(r, y)=\frac{1}{n} \sum_{i=1}^{n}\left[g\left(\left|\bar{\lambda}_{j}\right|-\frac{1}{2}\right)-g\left(\left|\lambda_{j}^{\text {out }}\right|-\frac{1}{2}\right)\right] .
$$

Таким образом, для вычисления коэффициента передачи информации необходимо найти $n$ симплектических значений $\bar{\lambda}_{j}^{\text {out }}, \lambda_{j}^{\text {out }}$ для соответствующих матриц $\overline{\mathcal{V}}^{\text {out }}$ и $\mathcal{V}^{\text {out }}$.

Коэффициент передачи (38) был найден численно в некоторых случаях, когда $\epsilon=\theta=1$. Из рис. 1 следует, что если $s>0$, то коэффициент $R$, максимизированный по $y$, возрастает с ростом степени зацепления $r$ и достигает максимума при некотором значении $r^{*}>0$, так что максимум достигается при зацепленных входных состояниях и он выше, чем при $s=r=0$. 


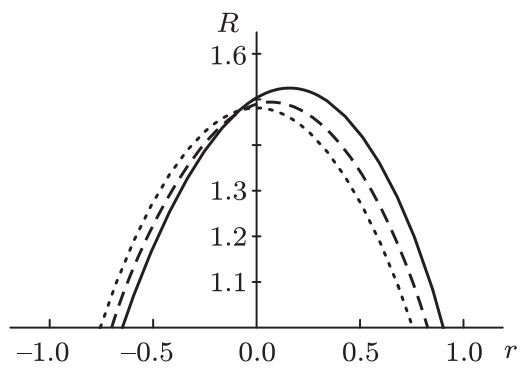

$a$

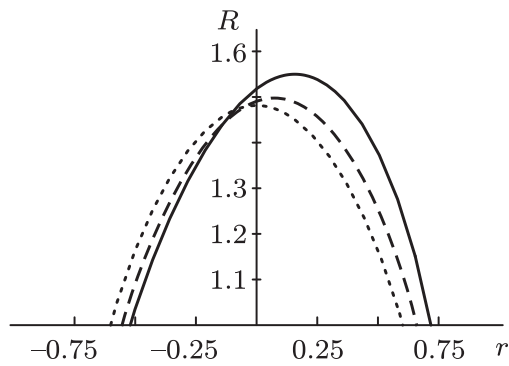

B
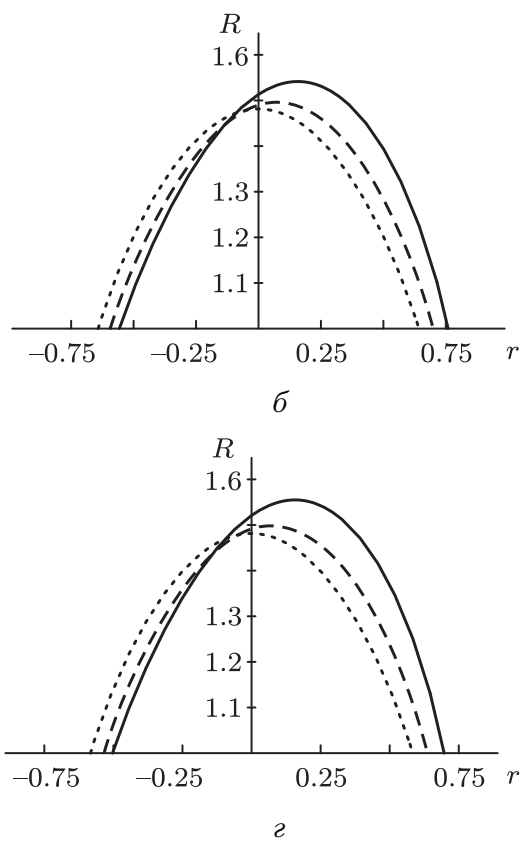

Рис. 1. Коэффициент передачи информации $R$ (максимизированный по коэффициенту $y$ ) как функция входного параметра зацепления $r$ для канала со степенью памяти $s=0$ (пунктирные кривые), $s=0.1$ (штриховые кривые) $s=0.2$ (сплошные кривые) при числе использований канала $n=2($ a), $n=$ 3 (б), $n=4$ (в), $n=5$ (г). Среднее число фотонов на входе равно $\bar{n}=2$, а число добавленных фотонов равно $N=2 / 3$.

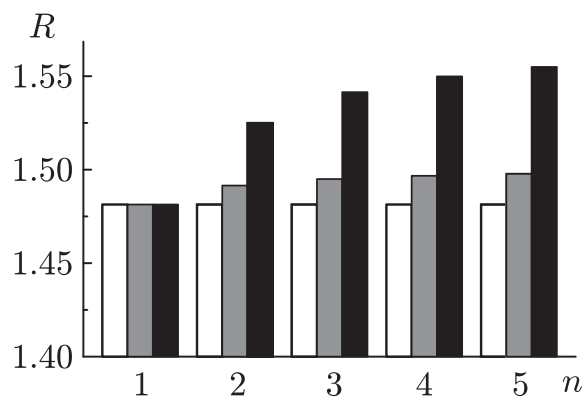

Рис. 2. Коэффициент передачи информации $R$ (максимизированный по параметру зацепления на входе $r$ и коэффициенту корреляции $y)$, представленный как функция от числа использований канала $n$ для канала со степенью памяти $s=0$ (белые прямоугольники), $s=0.1$ (серые прямоугольники) и $s=0.2$ (черные прямоугольники). Среднее число фотонов на входе $\bar{n}=2$, а число добавленных фотонов $N=2 / 3$. 
Более того, находя максимум $R$ как по $r$, так и по $y$, можно исследовать зависимость коэффициента передачи информации от числа использований канала $n$ (см. рис. 2). К сожалению, расчет $R$ при $n>5$ требует значительных вычислительных ресурсов. Тем не менее из рис. 2 следует, что коэффициент передачи информации возрастает с ростом числа использований канала $n$, стремясь к асимптотическому значению, зависящему от $s$. Это также можно понять из того, что коэффициент передачи возрастает с ростом шумовых корреляций. Они сначала быстро возрастают до тех пор, пока $n$ не достигает значения, совпадающего с числом эффективно взаимодействующих мод. На самом деле каждая мода эффективно взаимодействует с ограниченным числом других мод, которое задается (помимо вида $\mathcal{S}$ ) величиной $s$ (чем меньше $s$, тем меньше число эффективно взаимодействующих мод). Таким образом, число использований канала, после которого коэффициент $R$ становится почти постоянным, можно считать масштабом эффектов памяти.

\section{4. БОЗОННЫЙ КАНАЛ С ПОТЕРЯМИ}

Следуя работе [8], рассмотрим теперь бозонный канал с потерями (рис. 3) с коррелированным шумом, действующим на $n$ независимых мод электромагнитного поля, связанного с операторами уничтожения $\hat{a}_{j}, j=1,2, \ldots, n$. Каждый оператор $\hat{a}_{j}$ взаимодействует с модой окружения $\hat{b}_{j}$ с помощью разделителя пучка с передающей способностью $\eta \in[0,1]$, что моделирует потери.

Здесь входное состояние канала представляет собой смесь зацепленных когерентных состояний, определяемую как

$$
|\psi(\boldsymbol{\mu})\rangle=\widehat{S}_{a}(r)\left[\widehat{D}_{a_{n}}\left(\mu_{n}\right)|0\rangle_{a_{n}} \ldots \widehat{D}_{a_{1}}\left(\mu_{1}\right)|0\rangle_{a_{1}}\right]
$$

где $\widehat{D}_{a_{j}}\left(\mu_{j}\right)$ - оператор сдвига отдельно взятой $j$-й моды, отвечающий комплексному числу $\mu_{j}$, а через $\widehat{S}_{a}(r)$ обозначен $n$-модовый оператор сжатия, связанный с входными модами $\hat{a}_{j}$. Параметр $r$ представляет собой параметр зацепления между различными использованиями канала (значение $r=0$ соответствует отсутствию зацепления). Будем предполагать, что состояния $|\psi(\boldsymbol{\mu})\rangle$ распределены с весом, отвечающим гауссовому распределению вероятности

$$
P(\boldsymbol{\mu})=\prod_{j=1}^{n} P_{j}\left(\mu_{j}\right), \quad P_{j}\left(\mu_{j}\right) \equiv \frac{1}{\pi N} e^{-\left|\mu_{j}\right|^{2} / N},
$$

где $N$ - среднее число фотонов на одно использование канала. На практике ввиду зацепления эффективное среднее число фотонов на одно использование канала имеет вид $N_{\text {eff }}=N+\operatorname{sh}^{2} r$. При этом можно записать матрицу плотности входного состояния $\hat{\rho}_{\text {in }}$ в виде

$$
\hat{\rho}_{\text {in }}=\int d \boldsymbol{\mu} P(\boldsymbol{\mu})|\psi(\boldsymbol{\mu})\rangle\langle\psi(\boldsymbol{\mu})| .
$$

В соответствии с работой [6] введем корреляции между действиям окружения на различные использования канала с помощью $n$-модового сжатого вакуумного состояния

$$
\hat{\rho}_{\mathrm{env}}=\widehat{S}_{b}(s)\left[|0\rangle_{b_{n}} \ldots|0\rangle_{b_{1} b_{1}}\langle 0| \ldots b_{n}\langle 0|\right],
$$




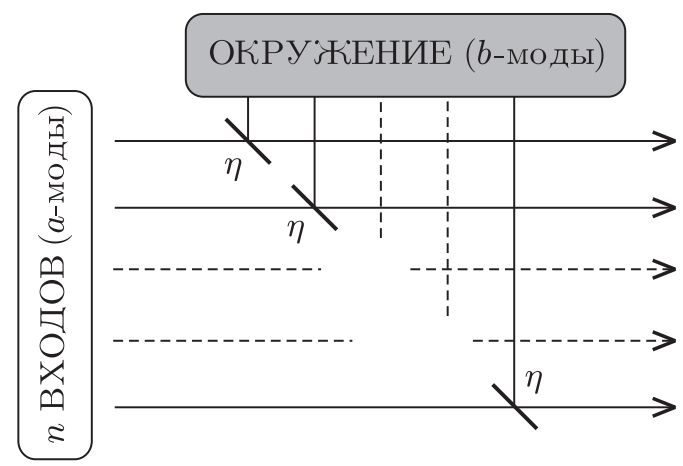

Рис. 3. Схема передачи информации: $n$ использований бозонного канала с потерями отвечают $n$ входным бозонным модам $\hat{a}_{j}$, взаимодействующим с модами окружения $\hat{b}_{j}$ посредством $n$ разделителей пучков.

где $\widehat{S}_{b}(s)$ представляет собой $n$-модовый оператор сжатия для мод окружения $\hat{b}_{j}$, a $s$ - параметр памяти, задающий эффекты корреляции шумов $(s=0$ отвечает случаю отсутствия памяти).

В нашей модели взаимодействие между входным состоянием и окружением задается преобразованием разделителя пучка [16]

$$
\hat{a}_{j} \longrightarrow \sqrt{\eta} \hat{a}_{j}-\sqrt{1-\eta} \hat{b}_{j}, \quad \hat{b}_{j} \longrightarrow \sqrt{\eta} \hat{b}_{j}+\sqrt{1-\eta} \hat{a}_{j} .
$$

Так как мы рассматриваем гауссовы состояния, оказывается полезным использовать функции распределения Вигнера [17]. Пусть $\hat{x}_{j}, \hat{p}_{j}$ - квадратурные переменные для $\hat{a}_{j}$, а $\mu_{j}^{\mathrm{R}}$ и $\mu_{j}^{\mathrm{I}}$ представляют собой вещественную и мнимую части $\mu_{j}$. Введем вектор-строки в $\mathbb{R}^{2 n}$

$$
\mathbf{u} \equiv\left[\hat{x}_{1}, \ldots, \hat{x}_{n}, \hat{p}_{1}, \ldots, \hat{p}_{n}\right], \quad \boldsymbol{\mu} \equiv\left[\mu_{1}^{\mathrm{R}}, \ldots, \mu_{n}^{\mathrm{R}}, \mu_{1}^{\mathrm{I}}, \ldots, \mu_{n}^{\mathrm{I}}\right]
$$

и вещественнозначную $(2 n \times 2 n)$-матрицу

$$
\mathcal{A}_{r}^{\prime}=\frac{2}{n}\left(\begin{array}{cc}
\mathcal{A}_{r}^{\prime \prime} & 0 \\
0 & \mathcal{A}_{-r}^{\prime \prime}
\end{array}\right)
$$

c $(n \times n)$-матрицей

$$
\mathcal{A}_{r}^{\prime \prime}=\left(\begin{array}{cccc}
e^{-2 r}+(n-1) e^{2 r} & e^{-2 r}-e^{2 r} & \cdots & e^{-2 r}-e^{2 r} \\
e^{-2 r}-e^{2 r} & e^{-2 r}+(n-1) e^{2 r} & \cdots & e^{-2 r}-e^{2 r} \\
\vdots & \vdots & \ddots & \vdots \\
e^{-2 r}-e^{2 r} & e^{-2 r}-e^{2 r} & \cdots & e^{-2 r}+(n-1) e^{2 r}
\end{array}\right) .
$$

Функция Вигнера, отвечающая состоянию на входе, имеет вид [17]

$$
W_{\text {in }}(\mathbf{u} ; \boldsymbol{\mu})=\left(\frac{2}{\pi}\right)^{n} \exp \left(-\mathbf{u} \mathcal{A}_{r}^{\prime} \mathbf{u}^{\mathrm{T}}-\boldsymbol{\mu} \mathcal{A}_{r}^{\prime} \boldsymbol{\mu}^{\mathrm{T}}+2 \boldsymbol{\mu} \mathcal{A}_{r}^{\prime} \mathbf{u}^{\mathrm{T}}\right) .
$$


Обозначим через $\mathbf{v} \equiv\left[\hat{y}_{1}, \ldots, \hat{y}_{n}, \hat{q}_{1}, \ldots, \hat{q}_{n}\right]$ вещественнозначный $2 n$-компонентный вектор квадратурных переменных $\hat{y}_{j}, \hat{q}_{j}$, связанных с операторами окружения $\hat{b}_{j}$. Тогда функция Вигнера, отвечающая $\hat{\rho}_{\text {env }}$, принимает вид

$$
W_{\text {env }}(\mathbf{v})=\left(\frac{2}{\pi}\right)^{n} \exp \left(-\mathbf{v} \mathcal{A}_{s}^{\prime} \mathbf{v}^{\mathrm{T}}\right)
$$

где матрица $\mathcal{A}_{s}^{\prime}$ задана выражением (45) при замене $r \rightarrow s$.

Определим векторы в $\mathbb{R}^{4 n}$ :

$$
\gamma \equiv[\mathbf{u}, \mathbf{v}], \quad \boldsymbol{\theta} \equiv[\mathbf{u}, \mathbf{0}], \quad \boldsymbol{\kappa} \equiv[\boldsymbol{\mu}, \mathbf{0}]
$$

Полную (т.е. учитывающую состояния на входе и эффекты памяти) функцию Вигнера можно представить в виде

$$
W_{\text {tot }}(\boldsymbol{\gamma} ; \boldsymbol{\kappa})=W_{\text {in }}(\mathbf{u} ; \boldsymbol{\mu}) W_{m}(\mathbf{v})=\left(\frac{2}{\pi}\right)^{2 n} \exp \left(-\boldsymbol{\gamma} \mathcal{A} \boldsymbol{\gamma}^{\mathrm{T}}+2 \boldsymbol{\kappa} \mathcal{A} \boldsymbol{\gamma}^{\mathrm{T}}-\boldsymbol{\kappa} \mathcal{A} \boldsymbol{\kappa}^{\mathrm{T}}\right),
$$

где $\mathcal{A}$ - вещественнозначная $(4 n \times 4 n)$-матрица вида

$$
\mathcal{A}=\left(\begin{array}{cc}
\mathcal{A}_{r}^{\prime} & 0 \\
0 & \mathcal{A}_{s}^{\prime}
\end{array}\right)
$$

Согласно (43) взаимодействие между сигналом и шумом отвечает замене переменных

$$
\gamma^{\mathrm{T}} \longrightarrow \mathcal{B} \gamma^{\mathrm{T}}
$$

производимой с помощью унитарной матрицы разделителя пучка

$$
\mathcal{B}=\left(\begin{array}{cc}
\sqrt{\eta} \mathcal{I} & \sqrt{1-\eta} \mathcal{I} \\
-\sqrt{1-\eta} \mathcal{I} & \sqrt{\eta} \mathcal{I}
\end{array}\right)
$$

где $\mathcal{I}$ - единичная матрица размера $2 n \times 2 n$. Сделав в (49) замену переменных (50), можно получить полную функцию Вигнера после взаимодействия между входными данными и окружением:

$$
W(\boldsymbol{\gamma} \boldsymbol{\kappa})=\left(\frac{2}{\pi}\right)^{2 n} \exp \left(-\gamma \mathcal{G} \boldsymbol{\gamma}^{\mathrm{T}}+2 \boldsymbol{\kappa} \mathcal{F} \boldsymbol{\gamma}^{\mathrm{T}}-\boldsymbol{\kappa} \mathcal{A} \boldsymbol{\kappa}^{\mathrm{T}}\right)
$$

где $\mathcal{F}=\mathcal{A B}$ и $\mathcal{G}=\mathcal{B}^{\mathrm{T}} \mathcal{A} \mathcal{B}$. Проинтегрировав (52) по переменным памяти $\mathbf{v}$, получим функцию Вигнера на выходе $W_{\text {out }}(\boldsymbol{\theta} ; \boldsymbol{\kappa})$.

Наконец, рассмотрим кодирование на выходе с помощью гетеродинного измерения, т.е. с помощью проекции матрицы плотности на выходе на $n$-модовое когерентное состояние $|\boldsymbol{\zeta}\rangle$. Теперь можно интерпретировать матричный элемент $\left\langle\boldsymbol{\zeta}\left|\hat{\rho}_{\text {out }}\right| \boldsymbol{\zeta}\right\rangle$ как условную вероятность, задаваемую явным выражением

$$
P(\boldsymbol{\zeta} \mid \boldsymbol{\mu})=\left(\frac{2}{\pi}\right)^{n} \int d \boldsymbol{\theta} W_{\text {out }}(\boldsymbol{\theta} ; \boldsymbol{\kappa}) \exp \left[-(\boldsymbol{\xi}-\boldsymbol{\theta}) \mathcal{L}(\boldsymbol{\xi}-\boldsymbol{\theta})^{\mathrm{T}}\right],
$$


где $\boldsymbol{\xi}$ - вещественнозначный $4 n$-компонентный вектор вида

$$
\boldsymbol{\xi} \equiv(\boldsymbol{\zeta}, \mathbf{0}), \quad \boldsymbol{\zeta} \equiv\left(\zeta_{1}^{\mathrm{R}}, \ldots, \zeta_{n}^{\mathrm{R}}, \zeta_{1}^{\mathrm{I}}, \ldots, \zeta_{n}^{\mathrm{I}}\right),
$$

а $\mathcal{L}-(4 n \times 4 n)$-матрица $\mathcal{L}=2\left(\begin{array}{cc}\mathcal{I}^{\prime} & 0 \\ 0 & 0\end{array}\right)$, в которой $\mathcal{I}^{\prime}-$ единичная $(2 n \times 2 n)$-матрица.

С помощью гауссовых интегралов условную вероятность (53) можно представить в виде

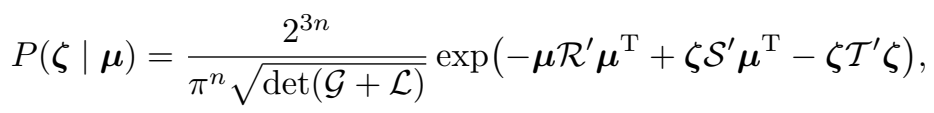

где $(2 n \times 2 n)$-матрицы $\mathcal{R}^{\prime}, \mathcal{S}^{\prime}$ и $\mathcal{T}^{\prime}$ представляют собой миноры, элементы которых лежат в первых $2 n$ строках и столбцах матриц

$$
\begin{aligned}
\mathcal{R} & =\mathcal{A}-\mathcal{F}(\mathcal{G}+\mathcal{L})^{-1} \mathcal{F}^{\mathrm{T}} \\
\mathcal{S} & =2 \mathcal{L}(\mathcal{G}+\mathcal{L})^{-1} \mathcal{F}^{\mathrm{T}} \\
\mathcal{T} & =\mathcal{L}-\mathcal{L}(\mathcal{G}+\mathcal{L})^{-1} \mathcal{L} .
\end{aligned}
$$

Соотношение (55) позволяет вывести также и совместную вероятность

$$
\begin{aligned}
& P(\boldsymbol{\zeta}, \boldsymbol{\mu}) \equiv P(\boldsymbol{\zeta} \mid \boldsymbol{\mu}) P(\boldsymbol{\mu})=\frac{2^{3 n}}{\pi^{2 n} N^{n} \sqrt{\operatorname{det}(\mathcal{G}+\mathcal{L})}} \times \\
& \times \exp \left[-\boldsymbol{\mu}\left(\mathcal{R}^{\prime}+\frac{\mathcal{I}^{\prime}}{N}\right) \boldsymbol{\mu}^{\mathrm{T}}+\boldsymbol{\zeta} \mathcal{S}^{\prime} \boldsymbol{\mu}^{\mathrm{T}}-\boldsymbol{\zeta} \mathcal{T}^{\prime} \boldsymbol{\zeta}^{\mathrm{T}}\right],
\end{aligned}
$$

равно как и вероятность на выходе

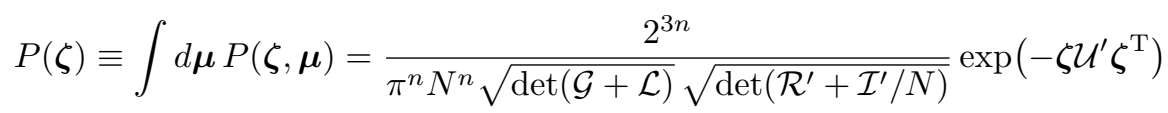

с вещественнозначной $(2 n \times 2 n)$-матрицей

$$
\mathcal{U}^{\prime}=\mathcal{T}^{\prime}-\frac{1}{4} \mathcal{S}^{\prime}\left(\mathcal{R}^{\prime}+\frac{\mathcal{I}^{\prime}}{N}\right)^{-1} \mathcal{S}^{\prime \mathrm{T}}
$$

Из определения энтропии Шеннона для вещественнозначной непрерывной стохастической переменной $\Phi$,

$$
I(\Phi)=-\int d \phi P(\phi) \log _{2} P(\phi)
$$

получим

$$
\begin{aligned}
I(\boldsymbol{\mu})= & \frac{n}{\ln 2}[1+\ln (\pi N)], \\
I(\boldsymbol{\zeta})= & \frac{2^{3 n}}{N^{n} \ln 2 \sqrt{\operatorname{det}(\mathcal{G}+\mathcal{L})} \sqrt{\operatorname{det}\left(\mathcal{R}^{\prime}+\mathcal{I}^{\prime} / N\right)} \sqrt{\operatorname{det\mathcal {U}^{\prime }}}} \times \\
& \times\left[n-\ln \left(\frac{2^{3 n}}{\pi^{n} N^{n} \sqrt{\operatorname{det}(\mathcal{G}+\mathcal{L})} \sqrt{\operatorname{det}\left(\mathcal{R}^{\prime}+\mathcal{I}^{\prime} / N\right)}}\right)\right]
\end{aligned}
$$




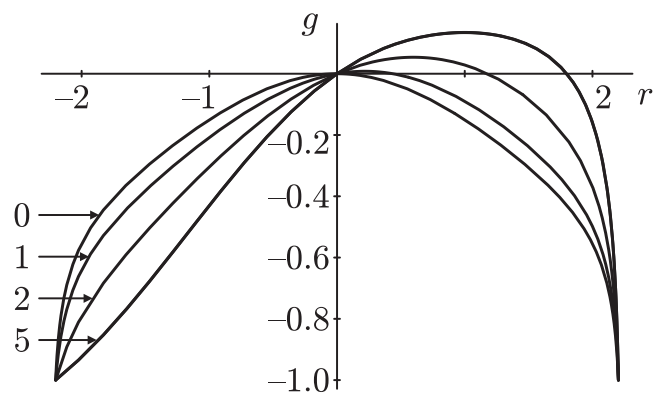

Рис. 4. Относительная переданная информация $g$ в зависимости от параметра зацепления $r$. Числа около кривых - значения степени памяти $s$. Значения остальных параметров выбраны равными $n=2, \eta=0.8$ и $N_{\text {eff }}=20$.

и

$$
I(\boldsymbol{\zeta}, \boldsymbol{\mu})=\frac{2^{3 n}}{N^{n} \ln 2 \sqrt{\operatorname{det}(\mathcal{G}+\mathcal{L})} \sqrt{\operatorname{det} \mathcal{V}}}\left[2 n-\ln \left(\frac{2^{3 n}}{\pi^{2 n} N^{n} \sqrt{\operatorname{det}(\mathcal{G}+\mathcal{L})}}\right)\right]
$$

c $(4 n \times 4 n)$-матрицей

$$
\mathcal{V}=\left(\begin{array}{cc}
\mathcal{R}^{\prime}+\mathcal{I}^{\prime} / N & -\mathcal{S}^{\prime} / 2 \\
-\mathcal{S}^{\prime} / 2 & \mathcal{T}^{\prime}
\end{array}\right)
$$

Теперь легко найти информацию, совместную для передатчика и приемника, т.е. совместную информацию, имеющую место при использовании вышеуказанной процедуры:

$$
I_{r} \equiv I(\boldsymbol{\zeta}: \boldsymbol{\mu})=I(\boldsymbol{\mu})+I(\boldsymbol{\zeta})-I(\boldsymbol{\zeta}, \boldsymbol{\mu})
$$

Напомним, что совместная информация, отнесенная к числу использований, т.е. $I_{r} / n$, задает коэффициент передачи информации. Таким образом, полезной величиной в нашем случае могла бы быть относительная переданная информация

$$
g=\frac{I_{r}-I_{r=0}}{I_{r=0}}
$$

которая характеризует полезность зацепленных входных данных.

Хотя мы и получили формальное выражение для совместной информации, почти невозможно сделать какие-либо выводы, анализируя это выражение. Поэтому величина $g$ была исследована численно как функция параметра зацепления $r$.

Поскольку мы намерены ограничить эффективное среднее число фотонов, приходящееся на каждое из использований канала, в реальных вычислениях фиксируется величина $N_{\text {eff }}$ как эффективное фотонное число на входе, а $N$ рассматривается как функция от $r\left(N=N_{\text {eff }}-\operatorname{sh}^{2} r\right)$, что ограничивает область определения $r$ на значения, при которых $N \geqslant 0$.

На рис. 4 показана относительная переданная информация $g$ в зависимости от параметра зацепления $r$ для различных значений степени памяти $s$ при двух использованиях канала и при фотонном числе на входе, равном $N_{\text {eff }}=20$. При $s=0$ 
график $g$ симметричен относительно точки $r=0$, в которой она достигает максимума $(g=0)$, т.е. зацепление входных данных бесполезно в случае отсутствия памяти. С ростом степени памяти симметрия нарушается, и при отрицательных значениях $r$ относительная переданная информация $g$ становится меньше, а при положительных - больше по сравнению со случаем $s=0$. В частности, в ограниченном интервале положительных значений $r$ она оказывается выше нуля. Отсюда с очевидностью вытекает, что максимум коэффициента передачи информации возрастает при зацеплении входных данных в случае каналов с памятью по сравнению с каналами без памяти $(g=0)$. Более того, существует оптимальное значение параметра $r$, зависящее от степени памяти, при котором $g$ принимает максимальное значение. Это более отчетливо проявляется при сильных эффектах памяти.

\section{5. ЗАКЛЮЧИТЕЛЬНЫЕ ЗАМЕЧАНИЯ}

В работе был исследован процесс передачи информации по непрерывным каналам в присутствии памяти.

В разделе 3 представлена модель, описывающая эффекты памяти в аддитивных гауссовых каналах при произвольном числе использований канала. Эта модель переходит в модель, описанную в работе [12], если ограничиться случаем двух использований канала связи. На основе полученных численных результатов выдвинуто предположение о существовании асимптотического значения для коэффициента передачи информации после многократного использования канала, которое должно отвечать наилучшей передающей способности канала. То есть знание передающей способности при двух использованиях оказывается недостаточным для того, чтобы охарактеризовать полную передаточную способность канала, если только эффекты памяти не слишком слабы. Можно сделать общее заключение, что минимальное число использований, которое оказывается достаточным для надежных заключений, зависит от степени памяти. Модель, которая используется в разделе 3 , может быть обобщена на случай, когда в расчет включаются эффекты затухания и усиления наряду с эффектами добавочных шумов.

В разделе 4 показано, что для рассматриваемой модели с каналом бозонной памяти с потерями и с непрерывным алфавитом, а также при некоторой выбранной процедуре кодирования (зацепленными состояниями) и декодирования (гетеродинными измерениями) можно заключить, что зацепление состояний можно использовать для повышения коэффициента совместной информации, по аналогии с тем, что происходит при рассмотрении некоторых классов каналов с дискретным алфавитом (см., например, работы [3], [4]).

Важно отметить, что указанная модель хорошо описывает физическую ситуацию, при которой все использования канала одинаково взаимно коррелированны. Это имеет место в случае, когда размер блоков таков, что эффект памяти один и тот же везде в блоке, а корреляция между различными блоками отсутствует. Однако эту модель можно легко приспособить и к исследованию целого ряда других ситуаций. 
Благодарности. Автор благодарна С. Манчини (University of Camerino) и Дж. Солиани (University del Salento) за сотрудничество при выполнении данной работы.

\section{Список литературы}

[1] M. A. Nielsen, I. L. Chuang, Quantum Computation and Quantum Information, Cambridge Univ. Press, Cambridge, 2000.

[2] J. Ball, A. Dragan, K. Banaszek, Phys. Rev. A, 69 (2004), 042324; K. Banaszek, A. Dragan, W. Wasilewski, C. Radzewicz, Phys. Rev. Lett., 92 (2004), 257901.

[3] C. Macchiavello, G. M. Palma, Phys. Rev. A, 65 (2002), 050301; C. Macchiavello, G. M. Palma, S. Virmani, Phys. Rev. A, 69 (2004), 010303(R).

[4] G. Bowen, S. Mancini, Phys. Rev. A, 69 (2004), 012306; G. Bowen, I. Devetak, S. Mancini, Phys. Rev. A, 71 (2005), 034310.

[5] R. G. Gallager, Information Theory and Reliable Communication, Wiley, New YorkLondon-Sydney-Toronto, 1968.

[6] V. Giovannetti, S. Mancini, Phys. Rev. A, 71 (2005), 062304.

[7] G. Ruggeri, S. Mancini, Quantum Gaussian channels with additive correlated classical noise, quant-ph/0609018.

[8] G. Ruggeri, G. Soliani, V. Giovannetti, S. Mancini, Europhys. Lett., 70 (2005), 719.

[9] D. Kretschmann, R.F. Werner, Quantum channels with memory, quant-ph/0502106.

[10] A. S. Holevo, M. Sohma, O. Hirota, Phys. Rev. A, 59 (1999), 1820.

[11] A. S. Holevo, R. F. Werner, Phys. Rev. A, 63 (2001), 032312.

[12] N. Cerf, J. Clavareau, C. Macchiavello, J. Roland, Phys. Rev. A, 72 (2005), 042330; Information transmission via entangled quantum states in Gaussian channels with memory, quant-ph/0508197.

[13] V. Giovannetti, S. Guha, S. Lloyd, L. Maccone, J. H. Shapiro, H. P. Yuen, Phys. Rev. Lett., 92 (2004), 027902.

[14] А. С. Холево, Вероятностные и статистические аспекты квантовой теории, Наука, M., 1980.

[15] C. F. Lo, R. Sollie, Phys. Rev. A, 47 (1993), 733.

[16] D. F. Walls, G. J. Milburn, Quantum Optics, Springer, Berlin, 1994.

[17] S. L. Braunstein, A.K. Pati (eds.), Quantum Information with Continuous Variables, Kluwer, Dordrecht, 2003. 